\title{
Erratum to: Hepatitis B in the United States: ongoing missed opportunities for hepatitis $B$ vaccination, evidence from the Behavioral Risk Factor Surveillance Survey, 2007
}

\author{
F. Ladak • A. Gjelsvik $\cdot$ E. Feller $\cdot$ S. R. Rosenthal $\cdot$ \\ B. T. Montague
}

Published online: 17 February 2012

(C) Springer-Verlag 2012

\section{Erratum to: Infection}

\section{DOI 10.1007/s15010-011-0241-2}

The correct affiliations of authors A. Gjelsvik and E. Feller are given below.

The author name S. Rosenthal should read S. R. Rosenthal.

The online version of the original article can be found under doi:10.1007/s15010-011-0241-2.

\section{F. Ladak}

Program in Public Health, Division of Biomedicine,

Brown University, 121 South Main Street,

Box G, Providence, RI 02903, USA

F. Ladak $(\square)$

72 Orchard Avenue, Barrington, RI 02806, USA

e-mail: farah.ladak@gmail.com

\section{A. Gjelsvik}

Program in Public Health, Brown University,

121 South Main St. S121-2, Providence, RI 02912, USA

e-mail: annie_gjelsvik@brown.edu

\section{E. Feller}

Department of Medicine, Alpert Medical School,

Brown University, 121 South Main St. S121-2,

Providence, RI 02912, USA

e-mail: Edward_feller@brown.edu

S. R. Rosenthal

Division of Biomedicine, Brown University,

121 South Main Street, Box G, Providence, RI 02903, USA

e-mail: samantha_rosenthal@brown.edu

B. T. Montague

Infectious Diseases, Brown University/Miriam Hospital,

164 Summit Ave, Providence, RI 02906, USA

e-mail: brianmontaguedo@gmail.com

\author{
A. Gjelsvik \\ Program in Public Health, Brown University, \\ 121 South Main St. S121-2, Providence, \\ RI 02912, USA \\ e-mail: annie_gjelsvik@ brown.edu \\ E. Feller \\ Department of Medicine, Alpert Medical School, \\ Brown University, 121 South Main St. S121-2, \\ Providence, RI 02912, USA \\ e-mail: Edward_feller@brown.edu
}

